\title{
Airway Management via Laryngeal Mask in Laryngotracheal Resection
}

\author{
Patrick Zardo ${ }^{1}$ Tom Kreft $^{2}$ Thomas Hachenberg ${ }^{2}$ \\ ${ }^{1}$ Department of Cardiothoracic Surgery, Otto von Guericke University, \\ Magdeburg, Germany \\ 2 Department of Anesthesiology and Intensive Care, Otto von Guericke \\ University Magdeburg, Germany \\ Address for correspondence Patrick Zardo, MD, Department of \\ Cardiothoracic Surgery, Otto von Guericke University, Leipziger Strasse \\ 44, Magdeburg 39120, Germany \\ (e-mail: patrick.zardo@med.ovgu.de).
}

Thorac Cardiovasc Surg Rep 2016;5:1-3.

\begin{abstract}
Keywords

- thoracic surgery

- airway management

- tracheal surgery

We present a case of impassable subglottic stenosis scheduled for tracheal resection and reconstruction managed by establishing a supraglottic airway. Despite careful preoperative evaluation, the stenosis was localized higher than anticipated, rendering conventional intubation impossible. Laryngeal mask bridging to cross-field ventilation was feasible and jet ventilation and cardiopulmonary bypass were available as emergency strategies. Surgery and emergence went uneventful. Perioperative considerations are discussed in this report.
\end{abstract}

\section{Introduction}

Despite technological advances in tracheal and tracheostomy tube design, postintubation tracheal stenosis (PITS) is still the predominant indication for tracheal resection. ${ }^{1}$ PITS is induced by cuff-associated submucosal compression, impaired regional blood flow, local cartilaginous ring ischemia, and secondary cicatrization. ${ }^{1}$ As most interventional procedures, including dilation ${ }^{2}$ and laser-therapy, ${ }^{3}$ almost inevitably lead to recurrence of tracheal stenosis, tracheal resection and reconstruction is generally considered the treatment of choice. $^{1-3}$ In most cases, initial mechanical ventilation can be achieved either through conventional tracheal intubation or jet ventilation. Alternative concepts are technically demanding and require complex multidisciplinary interaction, especially between the surgeon and anesthesiologist.

\section{Case Description}

A 53-year-old man in reasonable overall health $(1.75 \mathrm{~m} / 84 \mathrm{~kg})$ was referred to our department with a history of acute coronary syndrome, cardiopulmonary resuscitation, and percutaneous tracheostomy for extended mechanical ventilation over a period of 4 weeks 8 months before admission. He first reported episodes of exertion dyspnea, wheezing, and stridor
3 months after successful decannulation and mild PITS was diagnosed. One month later, an unrelated emergency neurosurgical procedure with a $6.5-\mathrm{mm}$ endotracheal tube (ETT) passing the stenosis and subsequent on-table extubation were described as uneventful despite bronchoscopically confirmed PITS. Because of continuously worsening exertion dyspnea, he underwent endoscopic laser therapy through rigid bronchoscopy under jet ventilation with good results 5 weeks after craniotomy. Unfortunately, his exertion dyspnea worsened over the next 2 months and he finally developed a high-grade tracheal stenosis which was barely passable by fiberoptic bronchoscopy and confirmed through a computed tomographic (CT) scan (-Fig. 1). Our workup at that point conclusively showed a short but high-grade stenosis located well below the cricoid cartilage at the level of the first thoracic vertebra. These findings were further corroborated by body plethysmographic analyses with a specific resistance of $10.25 \mathrm{kPa} \mathrm{s}$ (871\%) and a total resistance of $1.56 \mathrm{kPa} \mathrm{s} / \mathrm{L}$ (519\%) in accordance with an extrathoracic obstruction and a projected tracheal diameter of 6 to $7 \mathrm{~mm} .{ }^{4}$ We scheduled him for tracheal resection after ascertaining full operability.

Anesthesia was induced with an intravenous infusion of $0.2 \mu \mathrm{g} / \mathrm{kg} / \mathrm{min}$ remifentanil and a bolus dose of $1.2 \mathrm{mg} / \mathrm{kg}$ propofol. Sufficient bag mask ventilation was followed by muscle relaxation with $0.6 \mathrm{mg} / \mathrm{kg}$ rocuronium. Direct received

February 19, 2015

accepted after revision

May 7, 2015

published online

November 24, 2015
DOI http://dx.doi.org/

10.1055/s-0035-1556061. ISSN 2194-7635.
@ 2016 Georg Thieme Verlag KG
Stuttgart · New York

License terms

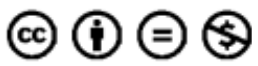




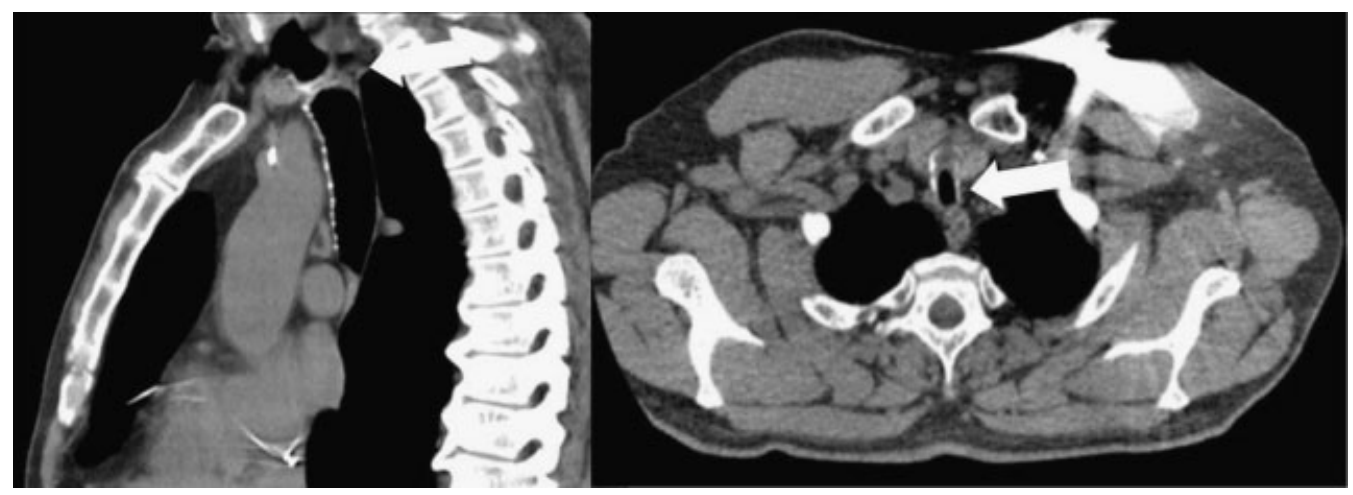

Fig. 1 Computed tomographic scan confirming the presence of a high-grade tracheal stenosis (arrow).

laryngoscopy confirmed Cormack-Lehane grade 1. Unexpectedly, the stenosis was localized in the upmost tracheal segment and had partial cricoid cartilage involvement, which effectively impeded a prestenotic tube placement. Neither 6.5- nor 6-mm ETT could pass the rigid stenosis in conventional or bronchoscopy-guided technique. Given the good intubation conditions and simple bag mask ventilation, we opted for supraglottic airway (SGA) management using a no. 5 laryngeal mask with jet ventilation and cardiopulmonary bypass which were readily available.

Anesthesia was continued as total intravenous anesthesia (TIVA) with remifentanil $0.2 \mu \mathrm{g} / \mathrm{kg} / \mathrm{min}$ and propofol $4 \mathrm{mg} / \mathrm{kg} /$ h. Pressure-controlled ventilation with low inspirational pressure was feasible with minimal leakage. Surgery was performed through a conventional low-collar incision and began with midline-centric dissection of the involved tracheal segments. After localizing the inferior stenosis margin and transecting the trachea, standard cross-field ventilation through a sterile $6.5-\mathrm{mm}$ tube was implemented (-Fig. 2). Because of cricoid involvement, a standard arcuate resection (anterior cricoid cartilage and cartilaginous lower tracheal segment) for laryngotracheal resection was performed. Reconstruction was achieved through lateral tension sutures (3/0 Vicryl, Ethicon/Johnson and Johnson Limited, Norderstedt, Germany) and a running suture (4/0 Maxon, Medtronic, Minneapolis, Minnesota, United States) for membranaceus reconstruction during cross-field ventilation. After switching to orotracheal intubation and placing the tube cuff distally to our involved field, interrupted sutures (2/0 Maxon) completed the cartilaginous aspect of the anastomosis. To avoid inadvertent neck overextension, a chin stitch was placed. Asleep extubation was followed by the bronchoscopic evaluation of anastomotic patency through a reinserted laryngeal mask. After discontinuation of anesthetic drugs and cough-free emergence, the patient was brought to our postanesthesia care unit. Postoperative recovery was uneventful and we could discharge the patient by postoperative day 7 after bronchoscopic control of the anastomosis.

\section{Discussion}

Airway management in tracheal surgery is generally demanding and requires an interdisciplinary approach. ${ }^{5} \mathrm{~A}$ meticulous preoperative workup, including CT scan (preferably with 3D reformation) and (rigid) bronchoscopy, facilitates all subsequent procedures.

In our case, Simplified Airway Risk Index evaluation and previous Cormack-Lehane 1 grading did not predict difficult intubation or bag mask ventilation. CT tracheal diameter, spirometry, and both previous rigid bronchoscopy and anesthesia for craniotomy predicted the passage of at least a 6.0$\mathrm{mm}$ ETT through a stenosis located well below the cricoid cartilage.

For induction of anesthesia, the patient should preferably assume an asymptomatic position. Preoxygenation may take longer than usual because of smaller tidal volumes. We encourage TIVA using propofol and remifentanil as it allows for rapid emergence and protection of the anastomosis because of reduced risks for postoperative nausea and vomiting. ${ }^{6}$ Even though inhalation anesthesia is potentially feasible in tracheal resection, ${ }^{7}$ workplace exposure to volatile anesthetics and interrupted drug supply during surgery-related apnoeic phases should be considered. A conventional intravenous induction should only be performed with reversible agents and after meticulous measurement of stenosis localization and diameter. An additional SGA problem potentially leading to a "cannot ventilate cannot intubate" situation has to be ruled out.

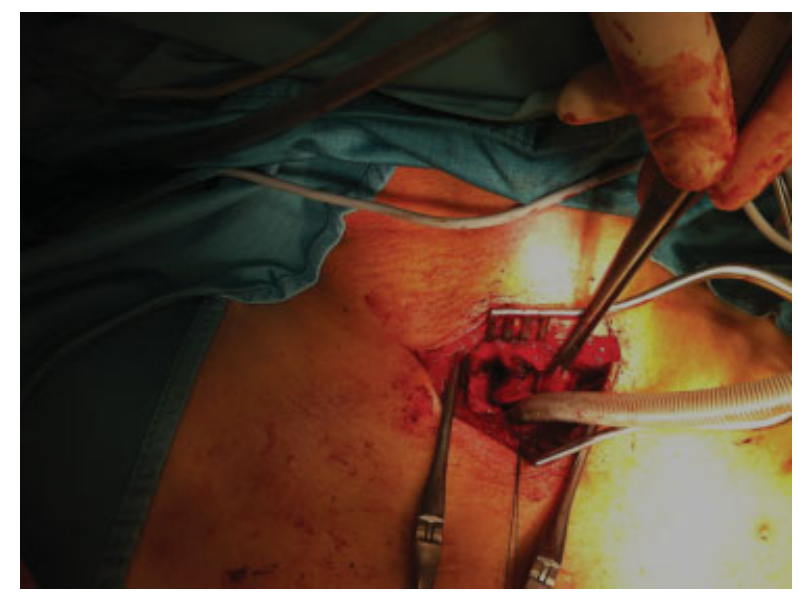

Fig. 2 Established cross-field ventilation with a sterile Woodbridge tube. 
Despite various randomized control trials suggesting a success rate of fiberoptic intubation in 88 to $100 \%$ of all patients with expected difficult airway and the technique being included in national difficult airway guidelines, ${ }^{8-10}$ most studies were based on patients with supraglottic causes for difficult airway, and conventional fiberoptic intubation may not be achievable in certain instances.

In our case, all previous examinations and interventions predicted easy bag mask ventilation and subglottic placement of an ETT of at least $6.0 \mathrm{~mm}$ diameter in direct laryngoscopy. With fallbacks in form of bag mask ventilation, SGA and jet ventilation readily available, we chose to perform a conventional induction with two attending anesthesiologists and a thoracic surgeon at the bedside.

Unexpectedly after intravenous induction, a rigid subglottic high-grade stenosis prevented intubation with a $6-\mathrm{mm}$ tube, and even fiberoptic inspection was barely feasible. To avoid swelling of the trachea due to manipulation, no further attempts at establishing airway control via conventional intubation were made. Instead we opted for a no. 5 laryngeal mask airway (LMA), thus committing to a SGA in accordance with published guidelines for difficult airway management. ${ }^{8-10}$ In case of impossible direct laryngoscopy the airway is to be secured via SGA, which in our department corresponds to LMA. Despite suggestions that LMA use should be preferably avoided whenever high inflation pressures are required, ${ }^{11}$ it has been sporadically reported in tracheal surgery, especially for pediatric patients, ${ }^{12}$ and constituted our best alternative. Definitive airway establishment would have been possible through railroading an ETT over a small diameter fiberoptic bronchoscope with or without dilation by gum elastic bougies or by using jet ventilation, for which a Monsoon jet device (Acutronic, Hirzel, Switzerland) was available. In our case, further manipulation and repeated attempts at bronchoscopy-guided passage might have aggravated the stenosis by a developing edema of vulnerable tracheal tissue, thus resulting in a "cannot ventilate cannot intubate" condition. Options for front of neck access, including awake cricothyrotomy or tracheotomy, were limited because of cricoid involvement and previous surgery. Implementation of venovenous extracorporeal membrane oxygenation can be potentially helpful in these circumstances and facilitate establishment of a safe distal airway, ${ }^{13}$ but was deemed unnecessary in this case.

After neuromuscular relaxation and under mandatory ventilation, laryngotracheal resection was initiated and reconstruction was performed under conventional cross-field intubation with a sterile $6.5-\mathrm{mm}$ tube according to standardized techniques. ${ }^{2}$

Subsequent intraoperative management needs to be geared toward on-table extubation to protect anastomoses from positive airway pressure and cuff-related injury as most postsurgical complications result from airway obstruction related to surgical field edema or blood clots. ${ }^{2,14}$ Stringent temperature management and perioperative pain therapy are essential to ensure an emergence without agitation, cough- ing, or shivering. At the end, a trans-LMA bronchoscopic control confirms anastomotic patency and simultaneously allows for bronchial rinsing from blood and/or secretions. Muscle relaxants should be reversed, as reintubation due to insufficient spontaneous breathing is challenging especially when a protective chin stitch is present and always endangers the anastomosis. We encourage utilization of cyclodextrins (sugammadex, Bridion, MSD, Haar, Germany) for safe and rapid reversal of muscle relaxation to avoid adverse effects by conventional antagonists. ${ }^{15}$ If inevitable, reintubation should be performed together with an attending thoracic surgeon.

In summary, airway management for tracheal surgery is demanding and requires a seamless multidisciplinary cooperation.

\section{References}

1 Wain JC Jr. Postintubation tracheal stenosis. Semin Thorac Cardiovasc Surg 2009;21(3):284-289

2 Grillo HC, Donahue DM. Postintubation tracheal stenosis. Chest Surg Clin N Am 1996;6(4):725-731

3 Pinsonneault C, Fortier J, Donati F. Tracheal resection and reconstruction. Can J Anaesth 1999;46(5, Pt 1):439-455

4 Empey DW. Assessment of upper airways obstruction. BMJ 1972; 3(5825):503-505

5 Blasberg JD, Wright CD. Surgical considerations in tracheal and carinal resection. Semin Cardiothorac Vasc Anesth 2012;16(4): 190-195

6 Donaldson AB, Meyer-Witting M, Roux A. Awake fibreoptic intubation under remifentanil and propofol target-controlled infusion. Anaesth Intensive Care 2002;30(1):93-95

7 Asai T, Shingu K. Airway management of a patient with tracheal stenosis for surgery in the prone position. Can J Anaesth 2004; 51(7):733-736

8 Apfelbaum JL, Hagberg CA, Caplan RA, et al; American Society of Anesthesiologists Task Force on Management of the Difficult Airway. Practice guidelines for management of the difficult airway: an updated report by the American Society of Anesthesiologists Task Force on Management of the Difficult Airway. Anesthesiology 2013;118(2):251-270

9 Henderson JJ, Popat MT, Latto IP, Pearce AC; Difficult Airway Society. Difficult Airway Society guidelines for management of the unanticipated difficult intubation. Anaesthesia 2004;59(7): 675-694

10 Braun U, Goldmann K, Hempel V, Krier C. Airway management. Leitlinien der Deutschen Gesellschaft für Anästhesiologie und Intensivmedizin. Anaesth Intensive Med 2004;45:302-306

11 Asai T, Morris S. The laryngeal mask airway: its features, effects and role. Can J Anaesth 1994;41(10):930-960

12 Asai T, Fujise K, Uchida M. Use of the laryngeal mask in a child with tracheal stenosis. Anesthesiology 1991;75(5):903-904

13 Ahn HY, Su Cho J, Kim YD, i H. Surgical outcomes of post intubational or post tracheostomy tracheal stenosis: report of 18 cases in single institution. Ann Thorac Cardiovasc Surg 2015;21(1):14-17

14 Piazza C, Del Bon F, Paderno A, et al. Complications after tracheal and cricotracheal resection and anastomosis for inflammatory and neoplastic stenoses. Ann Otol Rhinol Laryngol 2014;123(11): 798-804

15 Jahr JS, Miller JE, Hiruma J, Emaus K, You M, Meistelman C. Sugammadex: a scientific review including safety and efficacy, update on regulatory issues, and clinical use in Europe. Am J Ther 2015;22(4):288-297 